\title{
Structure and Magnetic Properties of a New Co" Thiophenedicarboxylate Showing Unprecedented Coordination
}

\author{
Aude Demessence, ${ }^{\dagger}$ Richard Welter, ${ }^{\dagger}$ Guillaume Rogez, ${ }^{\dagger}$ and Pierre Rabu ${ }^{*}, \dagger$ \\ Institut de Physique et Chimie des Matériaux de Strasbourg, UMR 7504 CNRS-ULP, 23 rue du Loess, BP 43, \\ 67034 Strasbourg cedex 2, France, \\ Laboratoire DECOMET, LC003 7177 CNRS-ULP ,Université Louis Pasteur Strasbourg I, F-67070 Strasbourg \\ cedex, France.
}

\section{Supplementary Materials}

\section{General remarks}

UV/Vis/NIR studies were performed on a Perkin-Elmer Lambda 19 instrument (spectra recorded by reflection with a resolution of $4 \mathrm{~nm}$ and a sampling rate of $240 \mathrm{~nm} \cdot \mathrm{min}^{-1}$ ). Thermogravimetric experiments were performed on a TA Instruments STD Q600 (heating in air from 20 to $700{ }^{\circ} \mathrm{C}$ at a rate of $5{ }^{\circ} \mathrm{C} \cdot \mathrm{min}^{-1}$ ).

Elemental analyses for C, H and S were carried out by the Analytical Department at the Institut Charles Sadron (Strasbourg, France). The determination of the quantity of Co was performed by oxidising pyrolysis [heating in air from $20^{\circ} \mathrm{C}$ to $900^{\circ} \mathrm{C}$ at $5^{\circ} \mathrm{C} / \mathrm{min}, 30 \mathrm{~min}$ at $700^{\circ} \mathrm{C}$ to transform into $\mathrm{Co}_{3} \mathrm{O}_{4}$ (the nature of the oxide was checked by X-ray powder diffraction) and cooling to $20^{\circ} \mathrm{C}$ at $\left.10^{\circ} \mathrm{C} / \mathrm{min}\right]$.

Magnetic data were collected with a SQUID magnetometer (Quantum Design MPMS-XL 5S) covering the temperature and field ranges $1.8-300 \mathrm{~K}, \pm 5 \mathrm{~T}$. 


\section{Synthesis}

Compound (1) is synthesized from $\mathrm{CoCl}_{2} \cdot 6 \mathrm{H}_{2} \mathrm{O}$ (98\% Aldrich) and thiophenedicarboxylic acid (99\% Aldrich) with sodium hydroxide in water. The starting mixture corresponding to the molar composition 1:1:2:1300 of $\mathrm{CoCl}_{2} \cdot 6 \mathrm{H}_{2} \mathrm{O}, \mathrm{C}_{6} \mathrm{H}_{4} \mathrm{SO}_{4}, \mathrm{NaOH}, \mathrm{H}_{2} \mathrm{O}$ is homogenized in a sealed hydrothermal bomb $\left(125 \mathrm{~cm}^{3}\right.$ bomb volume) walled with a Teflon liner under argon atmosphere and heated at $180^{\circ} \mathrm{C}$ during $22 \mathrm{~h}$. The resulting product consists of dark pink crystals, which are washed and rinsed with water and ethyl alcohol.

Anal. (\%) for $\mathrm{CoC}_{6} \mathrm{H}_{5} \mathrm{SO}_{5.5}, \mathrm{Mw}=256.10 \mathrm{~g} \cdot \mathrm{mol}^{-1}$ : found (calcd.): C 28.05 (28.14), H 1.96 (1.97), S 12.47 (12.52), Co 22.7 (23.0). 


\section{Crystal structure}

Single crystal of 1 was mounted on a Nonius Kappa-CCD area detector diffractometer (MoKa $=0.71073 \AA$ ). The cell parameters were determined from reflections taken from one set of 10 frames $\left(1.0^{\circ}\right.$ steps in phi angle), each at 20 seconds exposure. The structures were solved using direct methods and refined against $F^{2}$ using the SHELXL97 software (G.M. Sheldrick, SHELXL97, Program for the refinement of crystal structures; University of Gottingen, Germany, 1997).

Table S0 : Crystal data and refinement details for compound 1

\begin{tabular}{|c|c|}
\hline Formula & $\mathrm{C}_{6} \mathrm{H}_{5} \mathrm{CoO}_{5.5} \mathrm{~S}$ \\
\hline Formula weight & 256.09 \\
\hline Crystal system & monoclinic \\
\hline Space group & $\mathrm{C} 2 / \mathrm{c}$ \\
\hline $\mathrm{a}(\AA)$ & $19.8280(5)$ \\
\hline $\mathrm{b}(\AA)$ & $7.5130(2)$ \\
\hline c $(\AA)$ & $10.7300(3)$ \\
\hline alpha $\left(^{\circ}\right)$ & 90.00 \\
\hline Beta $\left(^{\circ}\right)$ & $103.440(2)$ \\
\hline gamma $\left({ }^{\circ}\right)$ & 90.00 \\
\hline $\mathrm{V}\left(\mathrm{A}^{3}\right)$ & $1554.65(7)$ \\
\hline $\mathrm{Z}$ & 8 \\
\hline Density $\left(\mathrm{g} \mathrm{cm}^{-3}\right)$ & 2.188 \\
\hline mu (Mo Kalpha) $\left(\mathrm{mm}^{-1}\right)$ & 2.466 \\
\hline $\mathrm{F}(000)$ & 1024 \\
\hline \multicolumn{2}{|l|}{ Data collection } \\
\hline Temperature $(\mathrm{K})$ & $173(2)$ \\
\hline Theta $\min -\max$ & $2.11-27.42$ \\
\hline Dataset[h, $\mathrm{k}, \mathrm{l}]$ & $-25 / 17,-8 / 9,-13 / 13$ \\
\hline Tot., Uniq. Data, R(int) & $1767,1595,0.0423$ \\
\hline Observed data & $>2 \operatorname{sigma}(\mathrm{I})$ \\
\hline \multicolumn{2}{|l|}{ Refinement } \\
\hline Nreflections, Nparameters & 1767,142 \\
\hline $\mathrm{R} 1, \mathrm{R} 2$ & $0.0258,0.0313$ \\
\hline wR1, wR2 & $0.0625,0.0649$ \\
\hline Goof & 1.086 \\
\hline Max. and Av. Shift/Error & $0.002,0.000$ \\
\hline Min, Max. Resd Dens. (e/A^3) & $-0.697,0.554$ \\
\hline
\end{tabular}


Table S1_A - Bond Distances (Angstrom)

\begin{tabular}{|c|c|c|c|c|c|}
\hline Co & -01 & $2.0275(14)$ & 03 & $-\mathrm{H} 4$ & $0.850(18)$ \\
\hline Co & -02 & $2.1128(13)$ & 03 & $-\mathrm{H} 3$ & $0.849(18)$ \\
\hline Co & -03 & $2.0959(15)$ & 07 & $-H 5 \_a$ & $0.85(2)$ \\
\hline Co & -05 & $2.0705(14)$ & 07 & $-\mathrm{H} 5$ & $0.85(2)$ \\
\hline Co & -07 & $2.1451(13)$ & C1 & $-\mathrm{C} 6$ & $1.470(3)$ \\
\hline Co & $-02 \_b$ & $2.1360(13)$ & $\mathrm{C} 2$ & $-\mathrm{C} 6$ & $1.370(3)$ \\
\hline$S$ & $-\mathrm{C} 4$ & $1.716(2)$ & $\mathrm{C} 2$ & $-\mathrm{C} 5$ & $1.407(3)$ \\
\hline$S$ & $-\mathrm{C} 6$ & $1.717(2)$ & $\mathrm{C} 3$ & $-\mathrm{C} 4$ & $1.478(3)$ \\
\hline 01 & $-\mathrm{C} 3$ & $1.258(2)$ & C4 & $-\mathrm{C} 5$ & $1.373(3)$ \\
\hline 02 & $-\mathrm{C} 1_{-} \mathrm{g}$ & $1.297(2)$ & $\mathrm{C} 2$ & $-\mathrm{H} 2$ & $0.92(2)$ \\
\hline 05 & $-\mathrm{CB}_{-} \mathrm{a}$ & $1.273(2)$ & $\mathrm{C} 5$ & $-\mathrm{H} 1$ & 0.92( \\
\hline 06 & $-\mathrm{C} 1$ & $1.247(2)$ & & & \\
\hline
\end{tabular}

Table S1_B - Bond Angles (Degrees)

\begin{tabular}{|c|c|c|c|c|c|c|c|}
\hline 01 & $-C_{0}$ & -02 & $169.01(6)$ & Co & -03 & $-\mathrm{H} 3$ & $130(2)$ \\
\hline 01 & $-C_{0}$ & -03 & $91.94(6)$ & Co & -07 & $-\mathrm{H} 5$ & $117(2)$ \\
\hline 01 & $-\mathrm{Co}_{0}$ & -05 & $100.32(6)$ & Co & -07 & $-\mathrm{H} 5 \_\mathrm{a}$ & $102.4(19)$ \\
\hline 01 & $-C_{0}$ & -07 & $88.25(5)$ & $\mathrm{H} 5$ & -07 & $-\mathrm{H} 5 \_\mathrm{a}$ & $105(2)$ \\
\hline 01 & $-C_{0}$ & $-02 \_b$ & $87.25(5)$ & $\mathrm{Co}_{-} \mathrm{a}$ & -07 & $-\mathrm{H} 5 \_\mathrm{a}$ & $117(2)$ \\
\hline 02 & $-C_{0}$ & -03 & $88.08(6)$ & Co_a & -07 & $-\mathrm{H} 5$ & $102.4(19)$ \\
\hline 02 & $-C_{0}$ & -05 & $90.67(5)$ & 06 & $-\mathrm{C} 1$ & $-\mathrm{C} 6$ & $118.93(18)$ \\
\hline 02 & $-\mathrm{Co}_{0}$ & -07 & $91.37(5)$ & 02_h & $-\mathrm{Cl}$ & -06 & $123.87(19)$ \\
\hline 02 & $-C_{0}$ & $-02 \_b$ & $81.76(5)$ & 02_h & $-\mathrm{C} 1$ & $-\mathrm{C} 6$ & $117.20(17)$ \\
\hline 03 & $-C_{0}$ & -05 & $89.02(6)$ & $\mathrm{C5}$ & $-\mathrm{C} 2$ & $-\mathrm{C} 6$ & $112.09(18)$ \\
\hline 03 & $-C_{0}$ & -07 & $178.08(6)$ & 01 & $-\mathrm{C} 3$ & $-C 4$ & $117.01(17)$ \\
\hline 02_b & $-C_{0}$ & -03 & $88.99(6)$ & 01 & $-\mathrm{C} 3$ & $-05 \_a$ & $125.64(18)$ \\
\hline 05 & $-C_{0}$ & -07 & $92.83(5)$ & 05_a & $-C 3$ & $-C 4$ & $117.34(16)$ \\
\hline 02_b & $-\mathrm{Co}_{0}$ & -05 & $172.23(5)$ & $s$ & $-C 4$ & $-\mathrm{C5}$ & $112.08(15)$ \\
\hline 02_b & $-C_{0}$ & -07 & $89.11(5)$ & $\mathrm{C} 3$ & $-C 4$ & $-\mathrm{C5}$ & $128.47(18)$ \\
\hline C4 & $-S$ & $-\mathrm{C} 6$ & $90.94(10)$ & $\mathrm{s}$ & $-C 4$ & $-\mathrm{C3}$ & $119.35(14)$ \\
\hline Co & -01 & $-\mathrm{C} 3$ & $133.37(13)$ & $\mathrm{C} 2$ & $-\mathrm{C5}$ & $-C 4$ & $112.47(18)$ \\
\hline Co & -02 & $-C o \_b$ & $98.24(5)$ & $S$ & $-\mathrm{C} 6$ & $-\mathrm{C2}$ & $112.42(15)$ \\
\hline Co & -02 & $-C 1_{-} \mathrm{g}$ & $122.35(13)$ & $\mathrm{C} 1$ & $-\mathrm{C} 6$ & $-\mathrm{C} 2$ & $129.42(18)$ \\
\hline Co_b & -02 & $-C 1_{-} \mathrm{g}$ & $118.29(12)$ & $S$ & $-\mathrm{C} 6$ & $-\mathrm{C} 1$ & $118.17(14)$ \\
\hline Co & -05 & $-C 3 \_a$ & $130.22(12)$ & $\mathrm{C} 5$ & $-C 2$ & $-\mathrm{H} 2$ & $127.1(15)$ \\
\hline Co & -07 & $-\mathrm{Co}_{-} \mathrm{a}$ & $113.25(10)$ & $\mathrm{C} 6$ & $-\mathrm{C} 2$ & $-\mathrm{H} 2$ & $120.8(15)$ \\
\hline Co & -03 & $-\mathrm{H} 4$ & $110.2(15)$ & $\mathrm{C} 2$ & $-C 5$ & $-\mathrm{H} 1$ & $126.7(14)$ \\
\hline H3 & -03 & $-\mathrm{H} 4$ & $106(3)$ & C4 & $-\mathrm{C5}$ & $-\mathrm{H} 1$ & $120.8(14)$ \\
\hline
\end{tabular}


Hydrogen bonds detected (Platon software) in compound 1.

$\begin{array}{lllll}\text { Donor -- H..Acceptor } & \text { D - H }(\AA) & \text { H...A }(\AA) & \text { D...A D }(\AA) & \text { D...A D }\left(^{\circ}\right) \\ \mathrm{O}(3)--\mathrm{H}(3) . . \mathrm{O}(5) \_\$ 1 & 0.849(18) & 1.902(18) & 2.747(2) & 173(3) \\ \mathrm{O}(3)--\mathrm{H}(4) . . \mathrm{O}(6) \$ 22 & 0.850(18) & 2.10(2) & 2.813(2) & 142(3) \\ \mathrm{O}(7)---\mathrm{H}(5) . . \mathrm{O}(6) \$ \_3 & 0.85(2) & 1.83(2) & 2.642(2) & 161(3)\end{array}$

Equivalent Position Code

$\$ 2=-1 / 2+x, 3 / 2-y,-1 / 2+z$

$\$ \_1=1-\mathrm{x}, 1-\mathrm{y}, 1-\mathrm{z}$

$\$ \_3=-1 / 2+x, 1 / 2+y, z$

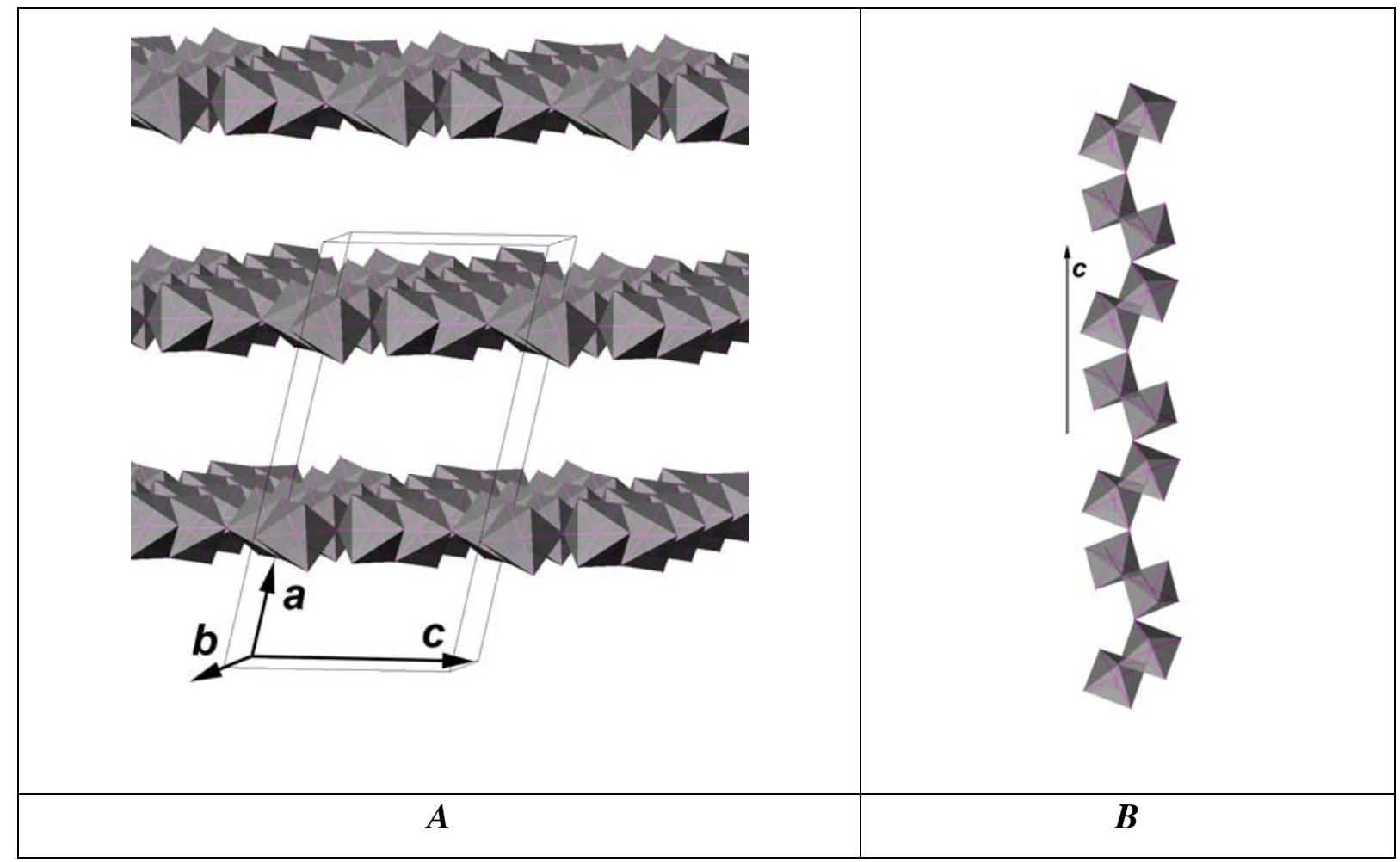

Figure S1. (A) Space filling polyhedra view of compound 1. The organic part has been omitted for clarity. (B) View of one chain of $\mathrm{CoO}_{6}$ polyedra along the crystallographic c-axis. 
As shown in Figure S2, the thiophenecarboxylates between chains form pairs in which the thiophene cycles are $\pi$-stacked at $3.62 \AA$. The pairs interact in a $\mathrm{T}$ configuration as usual for electron rich systems.

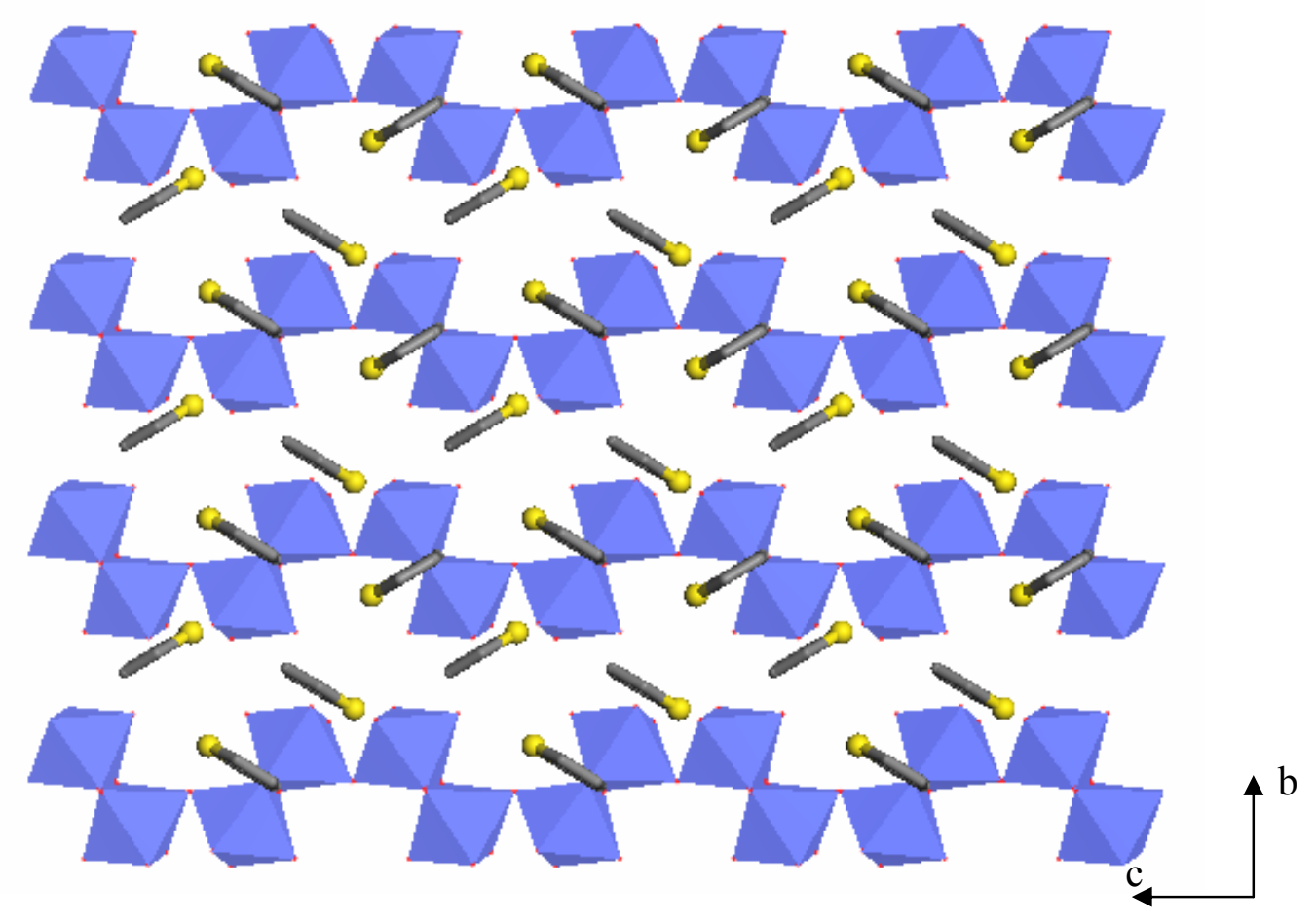

Figure S2. View of the chains of cobalt dimers in the (b,c) plane. The water molecules and carboxylate functions are omitted to underline the $\pi$ interactions between the thiophene rings. 


\section{Infra-red spectroscopy}

FT-IR studies were carried out with a Digilab Excalibur 3000 computer-driven instrument (0.1 mm thick powder samples in $\mathrm{KBr}$ ).

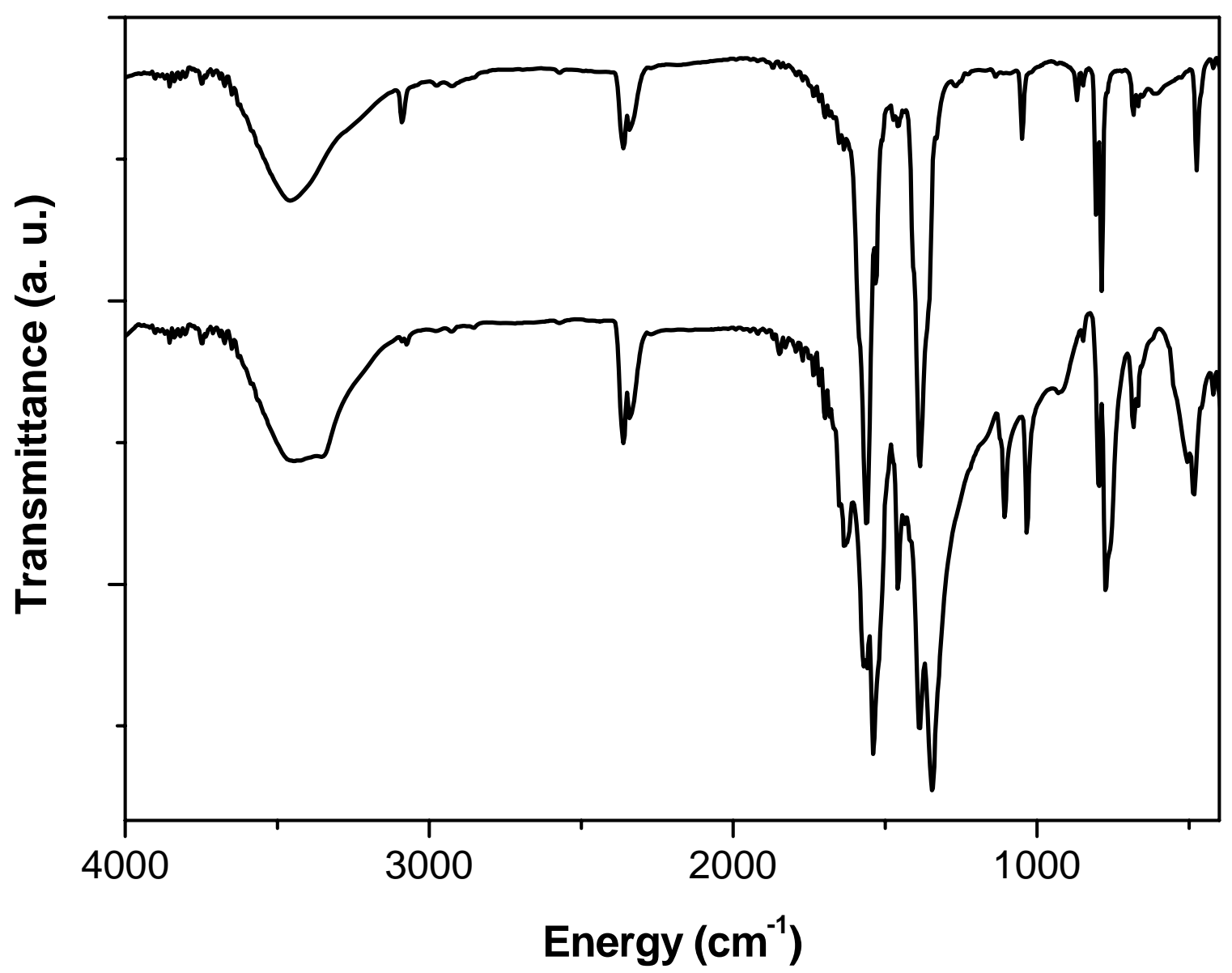

Figure S3. Infrared spectra of $\mathrm{Na}_{2} \mathrm{TDC}$ (top) and $\mathbf{1}$ (bottom).

Table S2. Selected vibrations for $\mathrm{Na}_{2} \mathrm{TDC}$ and $\mathbf{1 .}$

\begin{tabular}{|c|c|c|c|c|c|c|c|c|}
\hline & $\mathbf{v} \mathrm{H}_{2} \mathrm{O}$ & C-H & $\delta \mathbf{H}_{2} \mathrm{O}$ & $v_{\mathrm{as}} \mathrm{COO}$ & $\omega_{\text {noy }}$ & $\omega_{\text {noy }}$ & $v_{s} \mathrm{COO}$ & $\Delta v$ \\
\hline $\mathrm{Na}_{2} \mathrm{TDC}$ & 3460 & 3090 & - & 1560 & $\begin{array}{c}1530 \\
\text { (weak) }\end{array}$ & $\begin{array}{c}1475 \\
\text { (weak) } \\
1460 \\
\text { (weak) }\end{array}$ & 1385 & 175 \\
\hline 1 & 3400 & $\begin{array}{c}3090 \\
\text { (weak) } \\
3075 \\
\text { (weak) }\end{array}$ & 1630 & 1565 & 1540 & 1460 & $\begin{array}{l}1390 \\
1345\end{array}$ & $\begin{array}{l}175 \\
220\end{array}$ \\
\hline
\end{tabular}

$v$ : stretching, $\delta$ : bending, $\omega$ : in-plane ring vibration and $\Delta v=v_{\text {as }} \mathrm{COO}-v_{\mathrm{s}} \mathrm{COO}$. 


\section{$\underline{\text { UV-Vis. spectroscopy }}$}

UV/Vis/NIR studies were performed on a Perkin-Elmer Lambda 19 instrument (spectra recorded by reflection with a resolution of $4 \mathrm{~nm}$ and a sampling rate of $240 \mathrm{~nm} \cdot \mathrm{min}^{-1}$ ).

The solid state room-temperature UV-Vis. spectrum of $\mathbf{1}$ exhibit three $\mathrm{d}-\mathrm{d}$ transitions as expected for a $\mathrm{d}^{7}$ ion in octahedral symmetry in weak ligand field. The first band at $7500 \mathrm{~cm}^{-1}$ is attributed to the ${ }^{4} T_{1 g}(F) \rightarrow{ }^{4} T_{2 g}(F)$ transition, the second at $15000 \mathrm{~cm}^{-1}$ to the ${ }^{4} T_{1 g}(F) \rightarrow$ ${ }^{4} A_{2 g}(F)$ transition and the third at $19000 \mathrm{~cm}^{-1}$ to the ${ }^{4} T_{1 g}(F) \rightarrow{ }^{4} T_{1 g}(P)$ transition. From these observed transitions the Dq value and the Racah's parameter B are evaluated $\left(\mathrm{Dq}=860 \mathrm{~cm}^{-1}\right.$ and $\left.\mathrm{B}=840 \mathrm{~cm}^{-1}\right)$ and are in accordance with a weak ligand field $(\mathrm{Dq} / \mathrm{B} \approx 1)$ and a high-spin configuration for the metal centres. The weak nephelauxetic parameter, $\beta=0.75$, compared to $\beta\left(\mathrm{Co}\left(\mathrm{H}_{2} \mathrm{O}\right)_{6}{ }^{2+}\right)=0.85$, indicates a more covalent bond between the cobalt ions and oxygen atoms.

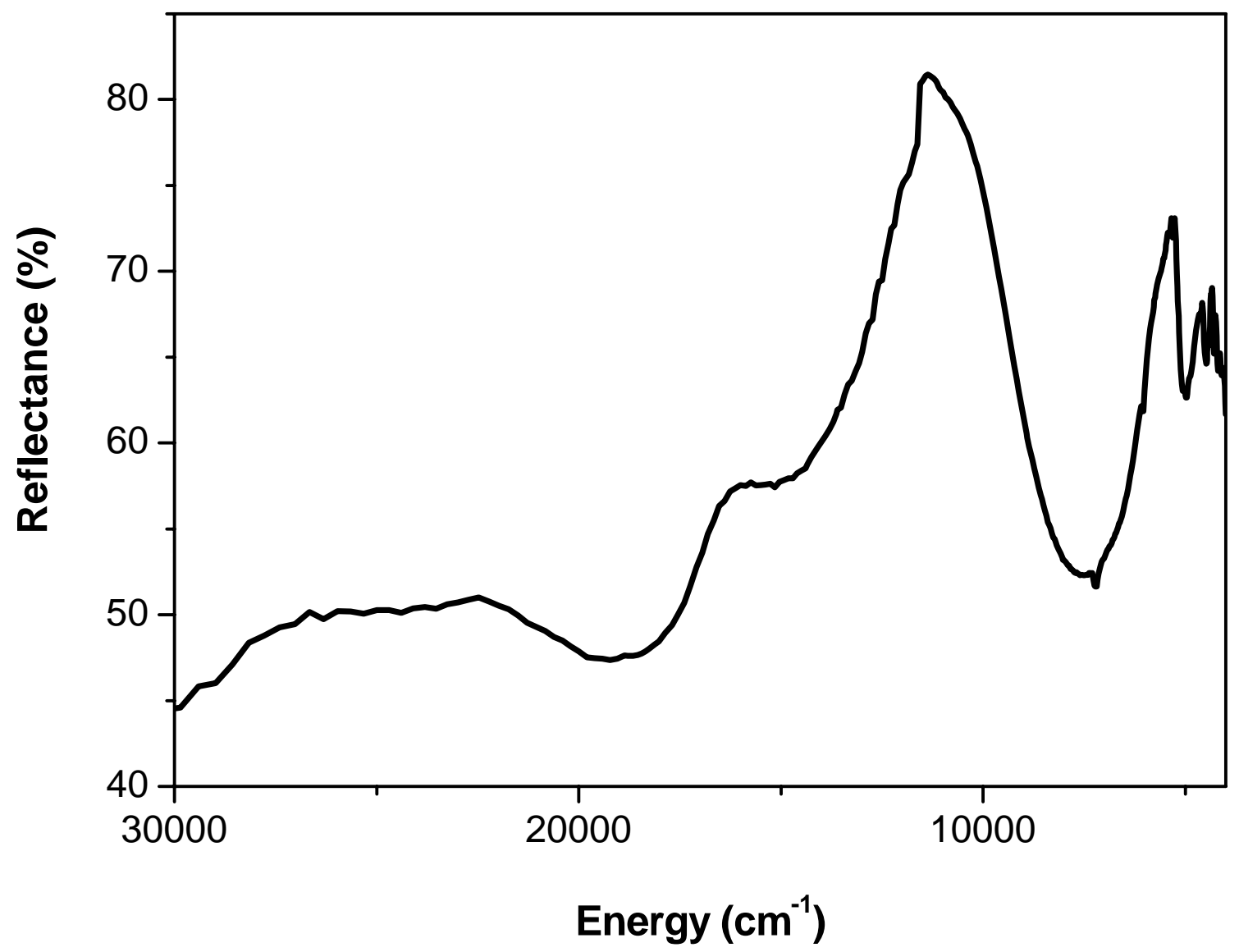

Figure S4. UV-Vis. reflectance spectrum of 1. 